\title{
REALIZATION OF PENTAEQUALIZER: 5.1 CHANNEL GRAPHIC EQUALIZER FOR IMPROVED SURROUND SOUND
}

\author{
Ninad Dileep Mehendale ${ }^{1}$ \\ ${ }^{I}$ Student, EXTC Department, K. J. Somaiya College of engineering, Maharashtra, India
}

\begin{abstract}
Home theaters and surround sound systems had become very popular in last few decades. Majority of these multi-channel systems are, without any channel graphic equalization and hence many time proves inefficient for sounds which are not recorded with multichannel mics. Pentaequalizer provide a combined hardware and software system that provide graphic equalizer for the sounds recorded with any platform (viz. Mono, stereo etc.). System has in-built mono to stereo and stereo to 5.1 channel audio conversion. This 5.1 channel graphic equalizer is named as pentaequalizer and it will be revolutionary innovation in terms of back compatibility of sound signals. The initial results show that improved SNR of 2.4dB and active noise reduction (ANR) of 6dB. Delay of the system varies from $1 \mathrm{~ms}$ to $3 \mathrm{~ms}$ depending upon type of audio input (e.g. Mono, Stereo and 5.1 Ch.)
\end{abstract}

Keywords: Pentaequalizer, Mono to stereo, Stereo to 5.1 Channel, and digital sound systems

\section{INTRODUCTION}

The split-surrounds also known as stereo is still the most popular music file format, because it has left and right channels, which are most suited for human hearing system specially when it comes to ear phones [1]. Digital 5.1 Ch. Dolby track consists of six analogous soundtracks [2]. The main advantage of $5.1 \mathrm{Ch}$. System over existing 2 channel system is that, it gives extra dimension making it more realistic. The first digital Dolby 5.1 surround sound was recorded back in 1975 for the film "Tommy" [3], since then over last few decades this multi-channel home surround sound has been the most popular choice of all music listening community. 5.1 channel system has 3 main channels with all frequencies, 2 high frequency channels and one low frequency bass channel. All X.1 systems has .1 as bass channel since $0.1 \mathrm{Khz}$ is about $100 \mathrm{~Hz}$ which is base frequency produced from many instruments like tabla, drums etc.

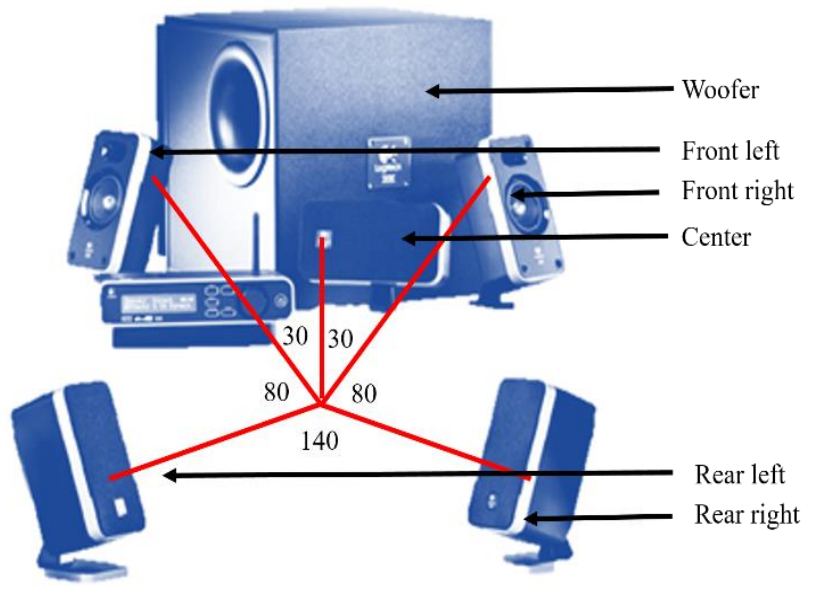

Fig -1: Typical configuration of 5.1 Ch. Surround sound system along with intra-speaker angles
The speaker arrangement of 5.1 Channel System is as shown in the fig -1, this is typical (but not a mandatory) arrangement of speakers. Woofer and central speaker are typically at $0^{\circ}$ angle to the listener. Front left and right are placed in line with center speaker and at angle of $30^{\circ}$, while rear speakers should be typically at $110^{\circ}$ from central speaker. Center where all red lines intersect is the position of listener.

\section{METHODOLOGY}

Pentaequalizer works with MATLAB code and actual hardware signal processing. System takes 2 types of inputs, first one from pre-recorded sound (such as MP3 or wav) or second could be live from microphone. For above inputs wavread, waverecord and audioreader command was used dependent on input type. As speed of sound is roughly 340 $\mathrm{m} / \mathrm{sec}$ and spacing between two ears is roughly $21 \mathrm{~cm}$ for adults hence delay of $\mathrm{D}$ seconds between two audio track (left and right) in case input is mono signal.

$$
\mathrm{D}=21 \times 10^{-2}(\mathrm{~m}) / 340(\mathrm{~m} / \mathrm{sec})=0.617 \mathrm{msec} .
$$

For conversion of mono to stereo we need only one delay, which is achieved in MATLAB by padding $\mathrm{N}$ zeros in beginning of second channel.

$$
\begin{gathered}
\mathrm{T}=1 / \mathrm{SR} \\
\mathrm{D}=\mathrm{N} \times \mathrm{T}
\end{gathered}
$$

Where:

$\mathrm{D}=$ Delay between two channels,

$\mathrm{T}=$ Time delay between two samples,

$\mathrm{N}=$ Number of zeros needed to pad before generated second channel from Mono channel input,

$\mathrm{SR}=$ Sampling rate (Number of samples recorded per unit time of one second.) 
Typical sample rate in audio is $44100 \mathrm{~Hz}$ hence time delay between two samples $\mathrm{T}=0.022 \mathrm{msec}$. Therefore, typical $\mathrm{N}$ comes out to be 27 zeros. This 27 number is only in case of $44100 \mathrm{~Hz}$ sampling frequency, but in audio recording this sampling rate vary from $8 \mathrm{KHz}$ to $96 \mathrm{KHz}$ and even $192 \mathrm{KHz}$ in some cases of high definition (HD) sound. After Mono to stereo (optional) conversion next step is to convert stereo signal to 5.1 channel signal. First for front right signal generation, original right soundtrack was filtered using $20 \mathrm{KHz}$ filter. Typically $\mathrm{y}=\operatorname{sgolayfilt}(\mathrm{x}$,order,framelen) is the MATLAB command used. This command applies Savitzky-Golay FIR smoothing filter to the audio signal data. Same operation was performed on original left soundtrack to generate front left channel.

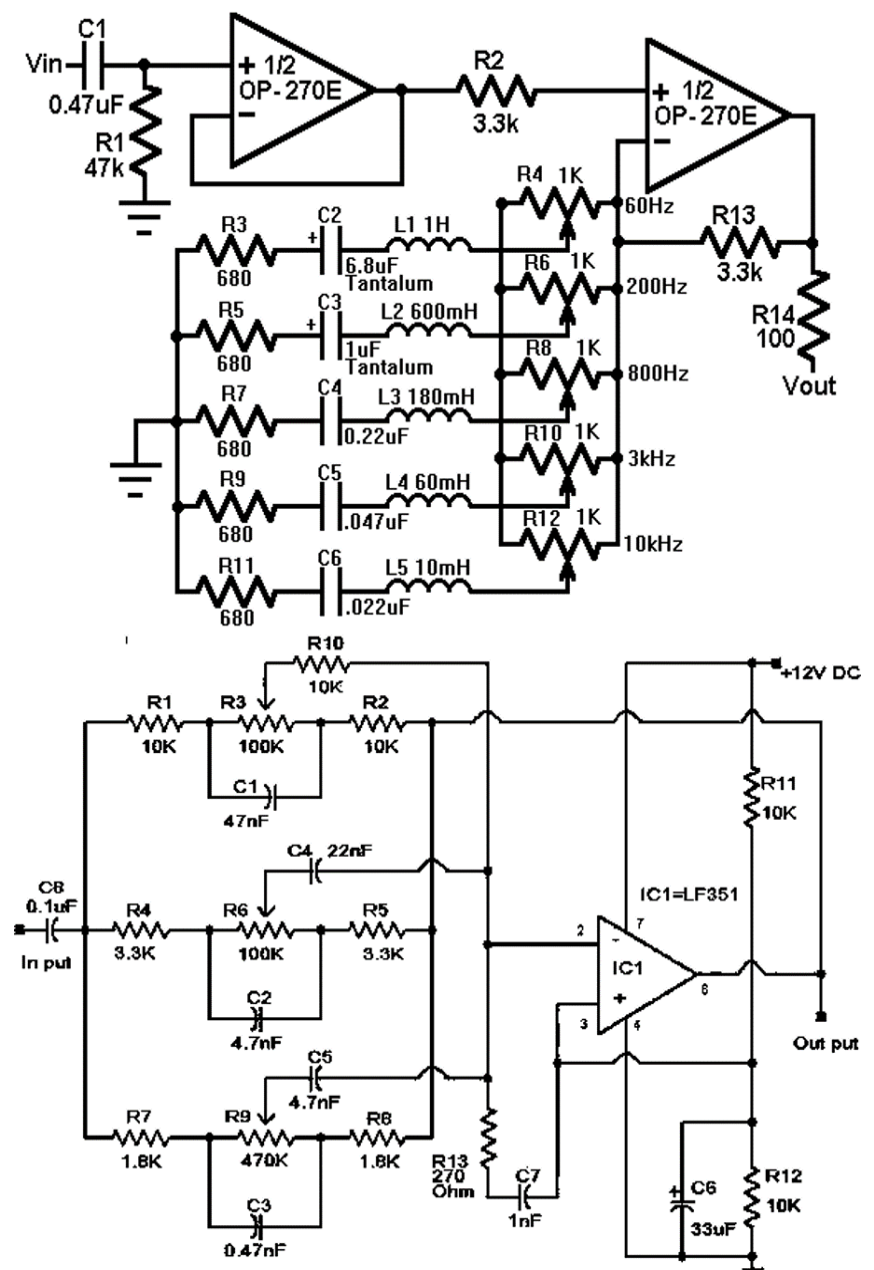

Fig -2: Circuit diagram of typical 5 band (Center, front left and right) and 3 band (woofer, rear left and right) graphic equalizer used for each channel.

For rear right channel the original right channel was filtered using high pass filter of $1 \mathrm{Khz}$. "[b,a] = cheby2(n,Rs,Ws,ftype)" is the command used in MATLAB. "ftype" was selected as "highpass" and cheyby2 was selected as it proves best for rear sound channel generation. Exactly same operation was repeated for rear left channel generation. For central channel generation stereo to mono is required. This channel was achieved by $\left(\mathrm{R}+\mathrm{L}_{\text {shift }}\right) / 2$, where $\mathrm{R}$ is original right channel and $\mathrm{L}_{\text {shift }}$ is phase shifted left channel. This is because simple average of two signals generally cancels out because they are $180^{\circ}$ out of phase signals. Unlike, karaoke generation, where user vocals are needed to be cancelled. Simple average works best for karaoke generation along with band reject filtering in range $1 \mathrm{KHz}$ to $4 \mathrm{KHz}$. Finally, $6^{\text {th }}$ channel for woofer was achieved by low pass filtering the central channel. MATLAB command for moving average filter $\mathrm{y}=$ filter $(\mathrm{b}, \mathrm{a}, \mathrm{x})$; proves to be the best one.

After using MATLAB coding all 5.1 channels were outputted through U-Mest PCI Express 5.1 PC Sound Card with 6 Channel Surround 3D audio output and uses CMI8738 chipset. Fig -2 shows circuit diagrams [4-5] for all channels. These standard circuits used simple Op-Amp amplifier filters designed with Op-Amp F351 and 270E. Regular Op-Amp do not work properly. Special audio amplifier Op-Amps are required for proper working of circuit. Typical values of RLC given in the circuit diagram is just a single case, but user can find values of RLC from frequency band for each of channel.

Typically, L is chosen because it is difficult to fabricate inductors with specific number of turns and gauge size. For air coil inductor

$$
\mathrm{L}(\mu \mathrm{H})=\mathrm{d}^{2} \times \mathrm{n}^{2} /(18 \mathrm{xd}+40 \mathrm{xl})
$$

Where:

$\mathrm{L}=$ Inductance

$\mathrm{d}=$ coil diameter in inches (Standard Wire Gauge)

$l=$ coil length in inches

$\mathrm{n}=$ number of turns

This formula gives and rough estimate of an inductance value. For RLC filters (fig-2 top), Capacitance $\mathrm{C}$ is given by

$$
\mathrm{C}=1 /(2 \pi \mathrm{f})^{2} \mathrm{~L}
$$

Where:

$\mathrm{C}=$ Capacitance

$\mathrm{f}=$ Resonant frequency

$\mathrm{L}=$ Inductance

And $\mathrm{R}$ value in RLC filter is decided by the current. Lower the value of $\mathrm{R}$, better is the output, but $\mathrm{R}$ cannot be zero as it will short circuit at resonance where $\mathrm{X}_{\mathrm{L}}=-\mathrm{X}_{\mathrm{C}}$

And finally for RC tuned filter (fig-2 bottom) resistance $\mathrm{R}$ is given by

$$
\mathrm{R}=1 /(2 \pi \mathrm{f}) \mathrm{C}
$$

Where:

$\mathrm{C}=$ Capacitance

$\mathrm{f}=$ Resonant frequency

$\mathrm{R}=$ Resistance

For center channel, front right and front left 5 band equalizer was used. For remaining channels 3 band equalizer was used. Once entire hardware and software was ready, 
pentaequalizer was tested on 24 mono channel, 301 stereo channel and 7 pre-recorded 5.1 channel sound files. For analysis purpose pentaequalizers each channel output was tested for frequency response using spectrum analyzer "Siglent SDS055" model. Also comparative study was done with existing Logitech 2.1 system, 5.1 channel system and 5.1 channel system with pentaequalizer. Three different parameters were measured namely signal to noise ratio (SNR), active noise reduction (ANR) and delay time between output and input using MATLAB run and time command. These values are measured with five standard known SNR, ANR test files.

$$
\begin{gathered}
\mathrm{SNR}=\mathrm{P}_{\text {signal }} / \mathrm{P}_{\text {noise }} \\
\mathrm{ANR}=\mathrm{P}_{\text {signal }} \times \mathrm{P}_{\text {noise }} / \mathrm{P}_{\text {signal }} \times \mathrm{P}_{\text {antinoise }}
\end{gathered}
$$

\section{RESULTS AND DISCUSSIONS}

As seen from Fig -3 woofer frequency response lies within range of $20 \mathrm{~Hz}$ to $900 \mathrm{~Hz}$. The center, front left, and front right have similar response. These 3 main channel sounds almost cover $20 \mathrm{~Hz}$ to $10 \mathrm{KHz}$. Rear frequency sound creates absorbance effect and hence lower frequencies are suppressed and their response gradually increases from $1 \mathrm{KHz}$ onwards. Fig -4 shows result of comparison of 3 different systems. In terms of delay 2.1 systems are best as no. of channels are less there are less number of samples require to handle. When compared to 5.1 system with and without pentaequalizer, SNR was improved by $2.4 \mathrm{~dB}$ and active noise reduction (ANR) was improved with $6 \mathrm{~dB}$ value after attaching pentaequalizer. The delay for pentaequalizer is maximum because of software and hardware combined approach. This delay ranges in 1 to $3 \mathrm{~ms}$.

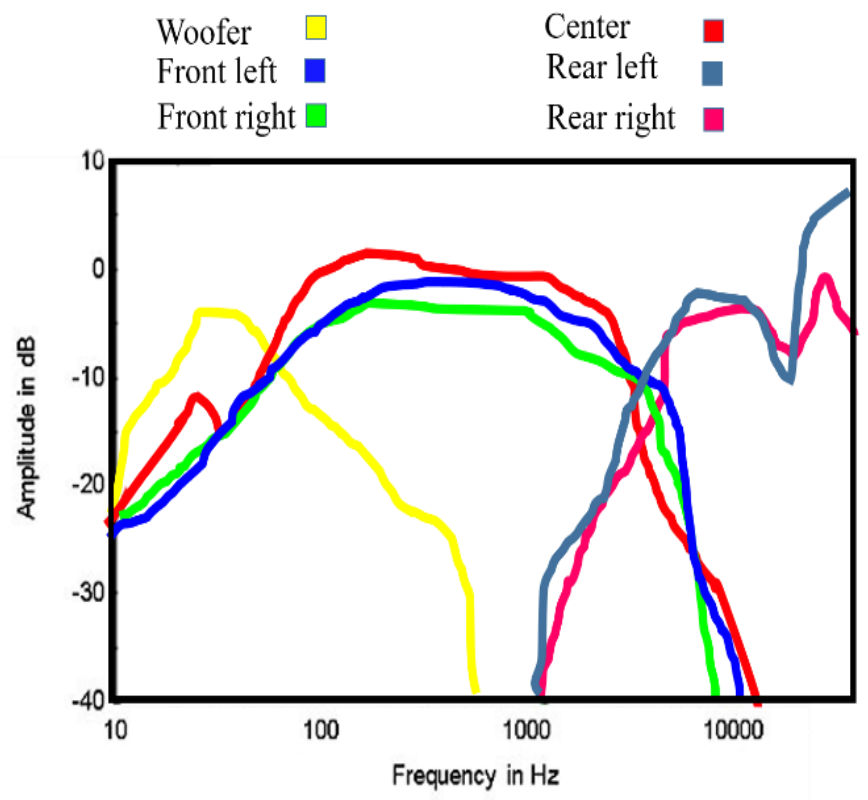

Fig -3: Measured frequency response of $5.1 \mathrm{Ch}$. Surround sound system for test sound "Time of your life.mp3"

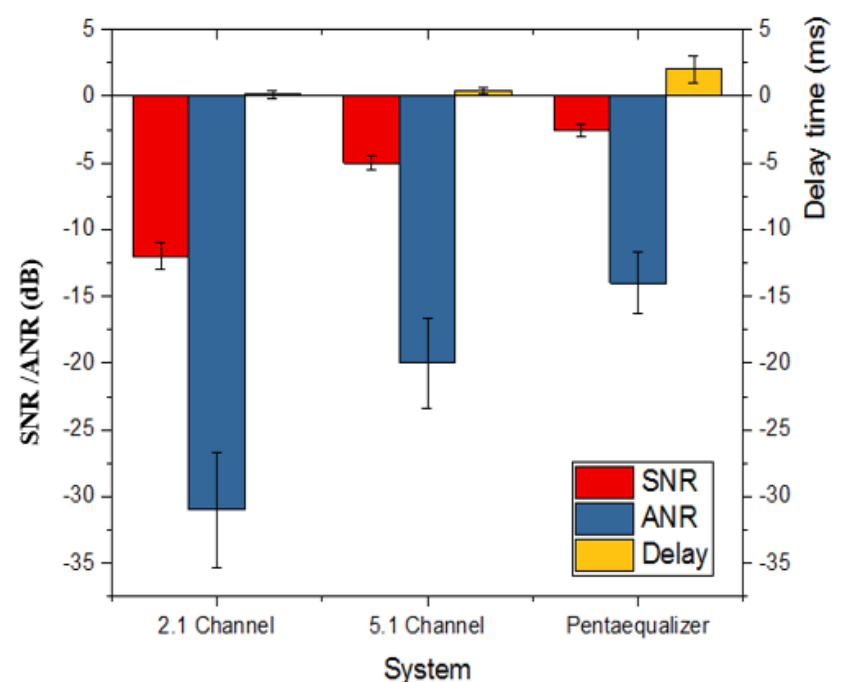

Fig -4: Comparison of 2.1 channel, 5.1 channel, and 5.1 channel attached with pentaequalizer system

\section{CONCLUSION}

Pentaequalizer is one of the most unique combination of hardware and software which converts any kind of input signal to 5.1 channel sound system. Currently system has very good SNR $(2.4 \mathrm{~dB})$ and ANR $(6 \mathrm{~dB})$ but suffers from issue of larger delay ( 1 - 3 milliseconds) because of software and hardware integration. This system can further be implemented on 7.1 channel sound or higher multi-channel home theater systems. Also the entire coding can be transferred from MATLAB to C program so that only hardware implementation is possible.

\section{ACKNOWLEDGEMENT}

The author would like to thank Swati Master, Abhijit Nayak, Milind Pathwardhan and project guide Prof. Swati Mahajan for support during initial phase of pentaequalizer. Author would also like to thank staff of K. J. Somaiya College of engineering for all the support and infrastructure.

\section{REFERENCES}

[1].Theile, Günther. "On the naturalness of two-channel stereo sound." Audio Engineering Society Conference: 9th International Conference: Television Sound Today and Tomorrow. Audio Engineering Society, 1991.

[2].Kerins, Mark. Beyond Dolby (stereo): cinema in the digital sound age. Indiana University Press, 2010.

[3].https://en.wikipedia.org/wiki/5.1_surround_sound

[4].http://www.circuitstoday.com/wp-content/uploads/2008/ 05/3-band-graphic-equalizer-circuit.jpg

[5].http://www.edaboard.com/thread124478.html

\section{BIOGRAPHIES}

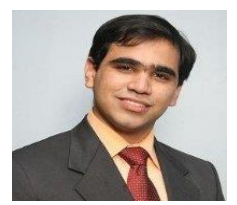

Mr. Ninad Dileep Mehendale is currently pursuing his $\mathrm{PhD}$ from Indian Institute of Technology Bombay, India. $\mathrm{He}$ has completed M-Tech and B.E. in Electronics and telecommunication. 